\title{
Budde, Jürgen
}

\section{Interaktionen im Klassenzimmer - Die Herstellung von Männlichkeit im Schulalltag}

Andresen, Sabine [Hrsg.]; Rendtorff, Barbara [Hrsg.]: Geschlechtertypisierungen im Kontext von Familie und Schule. Opladen : Verlag Barbara Budrich 2006, S. 113-119. - (Jahrbuch Frauen- und Geschlechterforschung in der Erziehungswissenschaft; 2)

Quellenangabe/ Reference:

Budde, Jürgen: Interaktionen im Klassenzimmer - Die Herstellung von Männlichkeit im Schulalltag In: Andresen, Sabine [Hrsg.]; Rendtorff, Barbara [Hrsg.]: Geschlechtertypisierungen im Kontext von Familie und Schule. Opladen : Verlag Barbara Budrich 2006, S. 113-119 - URN:

urn:nbn:de:0111-opus-53535 - DOI: 10.25656/01:5353

https://nbn-resolving.org/urn:nbn:de:0111-opus-53535

https://doi.org/10.25656/01:5353

in Kooperation mit / in cooperation with:

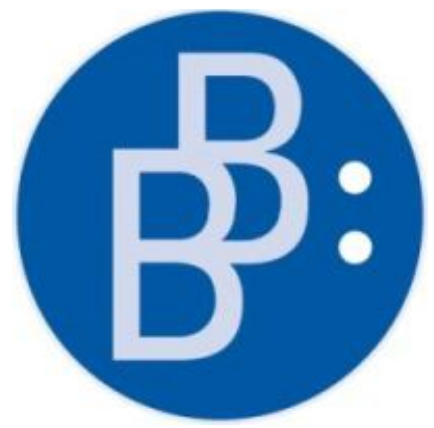

https://www.budrich.de

\section{Nutzungsbedingungen}

Gewährt wird ein nicht exklusives, nicht übertragbares, persönliches und beschränktes Recht auf Nutzung dieses Dokuments. Dieses Dokument ist ausschließlich für den persönlichen, nicht-kommerziellen Gebrauch bestimmt. Die Nutzung stellt keine Übertragung des Eigentumsrechts an diesem Dokument dar und gilt vorbehaltlich der folgenden Einschränkungen Auf sämtlichen Kopien dieses Dokuments müssen alle Urheberrechtshinweise und sonstigen Hinweise auf gesetzlichen Schutz beibehalten werden. Sie dürfen dieses Dokument nicht in irgendeiner Weise abändern, noch dürfen Sie dieses Dokument für öffentliche oder kommerzielle Zwecke vervielfältigen, öffentlich ausstellen, aufführen, vertreiben oder anderweitig nutzen.

Mit der Verwendung dieses Dokuments erkennen Sie die Nutzungsbedingungen an

\section{Kontakt / Contact:}

peDOCS

DIPF | Leibniz-Institut für Bildungsforschung und Bildungsinformation Informationszentrum (IZ) Bildung

E-Mail: pedocs@dipf.de

Internet: www.pedocs.de

\section{Terms of use}

We grant a non-exclusive, non-transferable, individual and limited right to using this document.

This document is solely intended for your personal, non-commercial use. Use of this document does not include any transfer of property rights and it is conditional to the following limitations: All of the copies of this documents must retain all copyright information and other information regarding legal protection. You are not allowed to alter this document in any way, to copy it for public or commercial purposes, to exhibit the document in public, to perform, distribute or otherwise use the document in public.

By using this particular document, you accept the above-stated conditions of use. 
Geschlechtertypisierungen im Kontext von Familie und Schule 
Jahrbuch der

Frauen- und Geschlechterforschung

in der Erziehungswissenschaft

herausgegeben von

Rita Casale

Barbara Rendtorff

Sabine Andresen

Vera Moser

Annedore Prengel

Beirat

Birgit Althans, Berlin

Eva Borst, Mainz

Eva Breitenbach, Osnabrück

Bettina Dausien, Bielefeld/München

Isabell Diehm, Bielefeld

Hannelore Faulstich-Wieland, Hamburg

Edgar Forster, Salzburg

Edith Glaser, Dortmund

Carola Iller, Heidelberg

Andrea Liesner, Hamburg

Susanne Maurer, Marburg

Inga Pinhard, Frankfurt

Folge 2/2006 
Sabine Andresen

Barbara Rendtorff (Hrsg.)

\section{Geschlechtertypisierungen im Kontext von \\ Familie und Schule}

Verlag Barbara Budrich, Opladen 2006 
Gedruckt auf säurefreiem und alterungsbeständigem Papier.

Die Deutsche Bibliothek - CIP-Einheitsaufnahme

Ein Titeldatensatz für die Publikation ist bei Der Deutschen Bibliothek erhältlich.

Alle Rechte vorbehalten.

(C) 2006 Verlag Barbara Budrich, Opladen

www.budrich-verlag.de

\section{ISBN 10: 3-86649-032-1}

ISBN 13: 978-3-86649-032-1

Das Werk einschließlich aller seiner Teile ist urheberrechtlich geschützt. Jede Verwertung außerhalb der engen Grenzen des Urheberrechtsgesetzes ist ohne Zustimmung des Verlages unzulässig und strafbar. Das gilt insbesondere für Vervielfältigungen, Übersetzungen, Mikroverfilmungen und die Einspeicherung und Verarbeitung in elektronischen Systemen.

Umschlaggestaltung: disegno visuelle kommunikation, Wuppertal - www.disenjo.de Satz: Susanne Rosenkranz, Opladen

Druck: DruckPartner Rübelmann, Hemsbach

Printed in Germany 
Inhalt

Editorial

\section{Essay}

Barbara Rendtorff

Von Müttern, Frauen und Schwindlerinnen. Anmerkungen zu Familie, Schule und Geschlecht - Ein Essay

\section{Beiträge}

\section{Marianne Friese}

Work-Life-Balance für junge Mütter. Neue Bildungsansätze und bildungspolitische Reformbedarfe zur Förderung von Kompetenz und Partizipation

Luitgard Franke

Demenz und Pflegebedürftigkeit in alten Paarbeziehungen -

ein Thema für die Geschlechterforschung

Rita Casale

Lebenslanges Lernen und die Erziehung der Frauen zu Müttern der Gesellschaft in der frühen Neuzeit

Astrid Messerschmidt

Aus dem Umgang mit der Geschichte lernen - Ansatzpunkte einer feministischen Kritik der Erinnerung in der dritten Generation nach dem Holocaust

\section{Work in progress}

Wolfgang Gippert

Nation und Geschlecht 
Sabine Toppe

Die Bedeutsamkeit von familiären Rollenbildern und

Geschlechtertypisierungen im Umgang mit Armut an Schulen

Jürgen Budde

Interaktionen im Klassenzimmer - Die Herstellung von Männlichkeit

im Schulalltag

Christine Hunner-Kreisel

Frauen und Religion in Aserbaidschan: Zwei Fallbeispiele

\section{Rezensionen}

Sabine Andresen

Rezension zu Edith Glaser/Dorle Klika/Annedore Prengel (Hg.):

Handbuch Gender und Erziehungswissenschaft, Bad Heilbrunn/OBB:

Verlag Julius Klinkhardt 2004

Myriam Rutschmann

Rezension zu Gertrud Hüwelmeier: Närrinnen Gottes.

Lebenswelten von Ordensfrauen, Münster/New York/München/Berlin:

Waxmann 2004

Sylvia Bürkler

Rezension zu Monika Simmel-Joachim/Reinhild Schäfer:

Frauenstudien zur Sozialen Arbeit. Wie genderbezogenes Wissen in die berufliche Praxis Eingang finden kann, Königstein/Taunus 2005

Susann Fegter

Rezension zu Barbara Rendtorff: Kindheit, Jugend und Geschlecht.

Einführung in die Psychologie der Geschlechter,

Weinheim/Basel/Berlin: Beltz Verlag 2003

\section{Ankündigung der nächsten Bände}

Jahrbuch Band 3

Jahrbuch Band 4 


\title{
Interaktionen im Klassenzimmer - Die Herstellung von Männlichkeit im Schulalltag
}

\author{
Jürgen Budde
}

\section{Einleitung}

Nachdem das deutsche Bildungssystem in den 1970er Jahren zu Recht als geschlechtsblind kritisiert wurde, gerieten zuerst Mädchen in den Fokus der Bemühungen einer geschlechtsbezogenen Pädagogik. Mit erheblichem Erfolg: galt früher das ,katholische Mädchen vom Lande“ als der Inbegriff der Bildungsbenachteiligten, so hat sich dieser Blick differenziert und gewandelt. Zunehmend häufiger stehen nun auch männliche Schüler im Zentrum pädagogischer Aktivitäten, meist im Zusammenhang mit Problemen. Durchschnittlich zeigen sie erstens geringere Bildungserfolge als ihre Mitschülerinnen und fallen zweitens häufiger durch schulunangepasstes Verhalten wie Stören oder Gewalt auf. Der neue Blick nimmt diese Probleme als geschlechtsbezogene Probleme wahr: Jungen erhalten als Jungen Beachtung.

Noch wenig beleuchtet ist der Anteil von Lehrkräften bei der Aushandlung von Männlichkeit im schulischen Alltag, insbesondere durch eigene diskrete stereotype Annahmen darüber, wie Jungen sind oder sein sollten ${ }^{1}$. Entgegen des eigenen Postulats der Gleichbehandlung werkeln auch Lehrkräfte häufig am , doing gender' mit.

Anhand empirischen Materials aus einer kürzlich abgeschlossenen Schulstudie thematisiert der Artikel zwei Aspekte. Zum ersten wird aufgezeigt, wie Schüler in Interaktionen mit Männlichkeitsstereotypen seitens der Lehrkräfte konfrontiert sind. Zum zweiten wird argumentiert, dass genau hieraus ein Dilemma erwächst, denn als ,typisch männlich“ etikettiere Eigenschaften sind in der Schule zunehmend weniger akzeptiert. Die daraus folgende These ist, dass Schüler vor dem Problem stehen können, sich entweder genderadäquat - und damit schuloppositionell - oder aber schuladäquat und damit genderunangepasst - zu verhalten.

Das Material stammt aus einer qualitativen Schulstudie, bei der drei Gymnasialklassen über drei Schuljahre der Sekundarstufe I hauptsächlich ethnographisch begleitet wurden (vgl. Faulstich-Wieland et. al. 2004, Budde 2005). Eine dieser Klassen war mit einem Verhältnis von 2/3 Jungen und 1/3 Mädchen jungendominant, eine zweite mit dem umgekehrten Verhältnis mädchendomi-

1 Auch Mädchen werden mit Genderstereotypen seitens der Lehrkräfte konfrontiert, allerdings in anderer Weise und mit anderen Konsequenzen (vgl. Budde/Faulstich-Wieland 2004). 
nant, die dritte ausgewogen zusammengesetzt. Insgesamt liegen annähernd 400 Stundenprotokolle (schwerpunktmäßig Deutsch, Mathematik, Englisch, Physik), ein Dutzend Interviews mit Lehrkräften, Gruppendiskussionen mit den Schülerinnen und Schülern sowie Fragebögen zu Selbsteinschätzung, Sachund Fachinteressen sowie zu Gleichberechtigung und Klassenklima vor.

Ralf Bohnsack betont, dass Ethnographie nicht in teilnehmender Beobachtung - die zweifellos ihr Herzstück darstellt - aufgeht. „Mit dem Begriff der Ethnographie wird nicht eine Methode bezeichnet, sondern ein Forschungsstil, der methodenplural angelegt ist" (Bohnsack 1997, S. 3). Man kann Ethnographie als Weg einer „Befremdung der eigenen Kultur“ sowie als „Erkenntnisstil [des] Entdeckens“" (Amann/Hirschauer 1997, S. 8) beschreiben. Auch Jürgen Zinnecker hebt auf die Befremdung ab, wenn er für die pädagogische Ethnographie sagt: „Es ist ein befremdender Blick, der auf die praxeologischen Selbstverständlichkeiten des Handelns und Wissens von Pädagogen und Kindern trifft und diese reflexiv verfügbar macht" (Zinnecker 1995, S. 51).

Ethnographischer Beobachtung stellt sich zwangläufig das Problem der Interpretation des Beobachteten durch die Forschenden im Beobachtungsund Verschriftlichungsprozess. Sowohl der subjektive Blickwinkel, als auch die Vorannahmen zur Genderthematik fließen in die erstellten Protokolle ein. Zugespitzt wurde dieses Problem in der vorliegenden Forschung noch dadurch, dass das Material nicht selber erhoben wurde, sondern auf ,fremderhobene“ Daten zurückgegriffen wurde. Zur Minimierung der Gefahr von ,Interpretationsunschärfe' wurden intensive Teamdiskussionen, Vergleiche mit ebenfalls erstellten Video- und Tonaufnahmen sowie gemeinsame Interpretationen von Protokollstellen im Gesamtforschungszusammenhang durchgeführt. Gleichzeitig bietet die Arbeit mit fremderhobenem Material in besonderer Weise die Möglichkeit des geforderten Befremdens gegenüber dem Beobachteten, da die Distanz zum Feld größer und die Gefahr, Beobachtetes für selbstverständlich zu halten, somit geringer ist. Für diesen Beitrag wurden Ankerbeispiele ausgewählt, die genderrelevante Mechanismen in den Interaktionen von Lehrkräften und Schülern besonders prägnant beleuchten.

\section{Interaktionen zwischen Lehrer und Schülern}

Auch wenn viel von den Veränderungen in der Genderordnung gesprochen wird, zeigt sich doch im Alltag, dass Männlichkeit nach wie vor eine zentrale Bezugs- und Identifizierungsgröße für die meisten Jungen darstellt. Das ,System hegemonialer Männlichkeiten‘ (vgl. Connell 1999, S. 87ff.) wirkt über einen Doppelmechanismus aus Konkurrenz und Solidarität, der dazu führt, dass einige ausgegrenzt werden, damit die anderen im Gegenzug inkludiert sind und ,dazugehören'. Beide Seiten widersprechen sich nicht, son- 
dern sind in der Aushandlung von Exklusion und Inklusion eine stabile Stütze von Männlichkeit (vgl. Budde 2003).

Für Ausgrenzungen werden unterschiedliche Schimpfworte und Unterstellungen verwendet: antisemitische oder rassistische Äußerungen, die Denunzierung von Mitschülern als ,behindert', kindlich etc. Symbolische Verweiblichungen stellen eine besonders dramatisierte Form dar. Das bedeutet, dass derjenige, der angeblich nicht der männlichen Norm entspricht, durch die Unterstellung von Homosexualität als unmännlich stigmatisiert wird. Die permanente Drohung, als schwul und somit als nicht-hegemonial zu gelten, erfordert geschlechtsadäquates Verhalten. Die ,Nicht-Hegemonialität' kann dann im Weiteren aufgrund der Dichotomie der Geschlechter als weiblich markiert werden. Ein wichtiger ,Resonanzboden' dafür ist die geschlechtshomogene Jungengruppe, welche die An- und Aberkennung von Männlichkeit gewährleistet bzw. verweigert (vgl. Budde 2005).

Viele Lehrkräfte sind dabei nicht geschlechtsneutral, sondern auf widersprüchliche Weise in diese Mechanismen involviert. Interaktionen sind dabei nicht die einzige, aber eine wichtige Ebene der Aushandlung von Gender, wie mit dem Begriff des „doing gender“ verdeutlicht wird. Rudolf Stichweh betont, dass Interaktionen als zentrale Vermittlungsmodi in Funktionssystemen wirken, die, wie die Schule, auf „professionelle Betreuungssituationen“ (2005, S. 21) ausgerichtet sind. Der Effekt von Interaktionen beschränkt sich dabei nicht nur auf die Beteiligten, sondern hat auch den Charakter einer Aufführung für die Beobachtenden, d.h. in diesem Falle für die MitschülerInnen (vgl. Beaufaÿs/Krais 2005). So werden in Interaktionen gemeinsame Deutungsfolien konstruiert.

Dies zeigt sich anhand eines Protokolls einer Klassenstunde der geschlechtsausgewogenen Klasse aus der Jahrgangsstufe 8. Es wird die Frage verhandelt, wann in der Doppelstunde die Pause stattfinden soll:

„Die Stunde geht dem Ende zu und Knut erkundigt sich, ob es eine Pause zwischen der 6. und 7. Stunde geben wird. Der Lehrer: ,Nein.' Knut fragt noch mal nach und Herr Bartoldi sagt: ,Schon, aber nicht zur offiziellen Pausenzeit.' Knut ist unzufrieden und mault. Nun fragt/sagt der Lehrer belustigt/argwöhnisch: ,Wieso? Nur weil du 'ne kleine Süße von nebenan treffen willst, soll die ganze Klasse warten?‘

Knut schweigt, die ganze Klasse schweigt, bevor viele SchülerInnen (bis auf Knut und Mark und einige andere) anfangen zu lachen und Knut necken. Knut bewegt sich nicht, Mark rückt sichtbar ein Stück näher an Knut ran und sieht ihn nicht an. Knut errötet. [...] Jemand ruft: ,Klein? Die ist größer als er.' Der Lehrer belustigt und extrem ironisch: ,Auch das noch. Eine größere?‘ [...]

Knut sagt noch immer nichts, inzwischen wird jedoch nach dem Namen gefragt. Jemand sagt: ,Ilka‘ Der Lehrer lacht und fragt nach: ,Ilka?' [...] Erik ruft, dass sie gar nicht Ilka heißt und viele unterhalten sich nun untereinander über Knut und seine Freundin. Als es klingelt, ist Knut erlöst.“ (By81111n)

Knuts Frage nach der Pause beantwortet der Lehrer mit einem ironischen Kommentar, indem er unterstellt, Knut wolle sich mit einer Freundin treffen. 
Die Protokollantin zitiert den Lehrer mit der zugleich entwertenden und sexualisierenden Beschreibung der vermeintlichen Freundin als ,'ne kleine Süße“. Durch die Ironie behält sein Kommentar zwar formal eine spaßhafte Ebene bei, gegen die sich Knut allerdings nur als ,Spaßverderber" positionieren könnte. Dies würde einen Bruch mit der Frauenabwertung von Herrn Bartoldi und damit ein Ausscheren aus dem System hegemonialer Männlichkeiten bedeuten - und zugleich riskante Opposition gegen einen Lehrer. Bei der Diskussion über die Körpergröße der Freundin führt Herr Bartoldi die Ironie durch die Kommentierung einer vermeintlichen ,Unnormalität ${ }^{6}$ fort: Wenn man als Junge mit einem Mädchen geht, so die implizite Aussage, dann muss diese kleiner sein. So etabliert der Lehrer gleich zwei Genderstereotype. Einerseits wird der Kontakt zwischen Jungen und Mädchen sexualisiert, zum zweiten normative Beziehungsstereotype festgeschrieben.

Mehrere Jugendliche greifen nach einer kurzen Phase des Schweigens und Zögerns die durch Herrn Bartoldi initiierte Ironie begeistert auf und setzen sie neckend durch Spekulationen über den Namen fort. Der Lehrer bestärkt so männersolidarisches Verhalten, dessen Grundlage hier blamierende Entwertung und sexualisierende Anspielung sind.

Auch bei anderen Kollegen lässt sich ein ähnlicher Mechanismus finden. Der Physiklehrer Herr Blümer wählt einen ,spaßhaften“ Vergleich mit dem Schüler Helmut, um zu erklären, wie der Begriff Leistung physikalisch definiert ist: „Also: Helmut rechnet 10 Matheaufgaben und er braucht ein Jahr [...] Ich mache 9 Aufgaben und brauche dafür einen Tag." Herr Blümer setzt sich selber an eine supremative Stelle und entwertet gleichzeitig Helmut. Auch hier zeigt sich die erwartbare Reaktion: „Die Klasse lacht schallend!“, gemeinsam mit dem Lehrer über Helmut. Die blamierende Markierung etabliert auf der einen Seite einen Außenseiter, auf der anderen Seite die komplizenhafte Jungengruppe. Hinter der Ironie des Lehrers verbirgt sich eine Mischung aus Entwertung und Komplizenschaft mit den Schülern der Klasse, welche sich gegen einen Jungen aus der Klasse richtet. Gegenüber Schülerinnen lässt sich diese Muster nicht in gleicher Weise finden.

Die beiden Lehrer sind somit an der Etablierung eines Klimas von Konkurrenz und Ironie beteiligt, welches anschlussfähig an Männlichkeitskonstruktionen ist und dem sich Knut und Helmut nicht entziehen können. Die beiden können sich weder auf der Ebene der Hierarchie, noch auf der Ebene von Männlichkeitskonstruktionen gegen die Lehrkräfte durchsetzen. Die „,komplizenhafte Männlichkeit“ (Connell 1999, S. 97) wird also auch in Interaktion zwischen Lehrkräften und Schülern hergestellt und gestärkt. Hier finden sich Sexualisierungen, Ironisierungen und hierarchische Solidarität als wichtige Elemente. Das männersolidarische Klima trägt so zu einer Fortführung des männlichen Habitus bei, der allerdings - wie im Folgenden gezeigt wird - nicht notwendigerweise ein Privileg darstellt. 


\section{Schüler zwischen Männlichkeit und Schule}

Gleichzeitig zeigen bspw. die PISA 2000-Studie, die hohe Zahl von Jungen an Haupt- und Förderschulen sowie die geringere Zahl von Abiturienten, dass zahlreiche Jungen im Bildungssystem nicht mehr so erfolgreich sind ${ }^{2}$. Eine Ursache wird in der „Feminisierung der Schule“ (Diefenbach/Klein 2001) gesehen. Allerdings greift diese These zu kurz, weil sie das Problem häufig personalisiert und auf die Frage der zahlenmäßigen Verteilung von Lehrerinnen und Lehrern reduziert (Stürzer et. al. 2003). Wesentlicher ist m.E. ein genereller Wertewandel in Bezug auf in der Schule honoriertes Verhalten. Tradierte Männlichkeit wird - wie oben beschrieben - in der Schule zwar erwartet und hergestellt. Parallel zeigt sich, dass genau dies zunehmend weniger positiv gewertet wird, sondern Jungen häufig negativ gesehen und so Stereotype festgeschrieben werden. Es besteht die Gefahr, dass die Jungen von Lehrkräften als geschlechtliche Gruppe homogenisiert werden, indem ihnen ,typisch männliche' Eigenschaften zugeschrieben werden.

Bereits die Charakterisierung der drei Klassen der Studie durch die Lehrkräfte zeigt einen deutlichen Rekurs auf negatives Jungenverhalten: Die jungendominante Klasse gilt als nicht ,normal ' gemessen am gymnasialen Standard. Viele Lehrkräfte stellen dabei einen unmittelbaren Zusammenhang zwischen der Jungendominanz und den Problemen mit der Klasse her - dies gilt ebenso für die vergleichsweise schlechteren Leistungen, für die Notwendigkeit, „,den Jungen mal zu zeigen, wer hier der Chef ist" ${ }^{\text {“3 }}$, sowie die Klage über die zu stillen und zurückhaltenden Mädchen. Diese Sicht auf die Schüler fließt auch in die Interaktionen ein und sorgt so für das oben beschriebene Dilemma.

Dazu ein Beispiel aus dem Englischunterricht der mädchendominanten Klasse im 8. Jahrgang. Die Lernenden nutzen eine von der Lehrerin vorgegebene sprachliche Übung dazu, sich gegenseitig wegen ihrer Frisuren zu necken. Die Lehrerin stellte die Aufgabe, sich zur Unterscheidung der Begriffe „since“ und „for“ gegenseitig Fragen zu stellen. Im Protokoll der Stunde heißt es:

„Nun ist Marianne dran. Sie sagt: ,Knut‘. Sie erntet Gelächter. Sie fragt: ,How long do you have ...' Zuerst fragt sie die Lehrerin, was schreckliche Frisur auf Englisch heißt. Lehrerin: ,Horrible hairdress'. Sie stellt nun an Knut die Frage: ,How long do you have this horrible hairdress?' Wieder großes Gelächter. Knut ironisch: ,I have this horrible hairstyle ...‘. Er setzt an, seine Nachbarin kommt ihm noch zuvor und sagt: ,Halbes Jahr'. Knut nickt zustimmend und sagt: ,For three months'. Er sagt: ,Marianne'. Die Kids lachen und neugierige Spannung entsteht, was seine Rückfrage ist.

2 Die PISA 2003-Studie zeigt allerdings ebenfalls, dass im mathematischen Bereich durchschnittlich Jungen leicht besser abschneiden und auch im sprachlichen Bereich eine ganze Reihe von Schülern sehr gute Ergebnisse erzielen.

3 So eine Aussage des Klassenlehrers. 
Er sagt: ,Ich weiß nicht ob das jetzt richtig ist: Since when do you have look like a horse?` Marianne, die einen langen Pferdeschwanz trägt, zuckt mit den Achseln, bevor sie jedoch irgendwie weiter reagieren kann, interveniert die Lehrerin. Sie schaut wieder zu Knut und sagt: ,We don't want insulting questions!‘ Knut sagt, leicht ironisch: ,Sie hat mich hier vorgeführt, so dass ich mich morgen nicht mehr in die Schule traue, und .... Die Lehrerin ironisch: ,Yes, I know, because you are so shy! “ “ (Be80928d)

Marianne und Knut nutzen die Aufgabenstellung, um sich über die Gendergrenzen hinweg zu necken, sehr zur Unterhaltung der Klasse - wie das Lachen deutlich zeigt. Die Lehrerin hilft Marianne bei der Suche nach der richtigen Vokabel, mit der sie Knut durch eine kritisierende Frage große Aufmerksamkeit entgegenbringen kann. Knut geht - auf gleicher spielerischer Ebene bleibend - darauf ein und kontert. Sein Beitrag jedoch wird von der Lehrerin schnell reglementiert und unterbunden, ebenso wie seine darauf folgende ironische Beschwerde. Er wird von der Lehrerin zurechtgewiesen, Marianne jedoch nicht, obwohl auch sie eine ironisch-blamierende Frage formuliert, bzw. die neckende Interaktion sogar beginnt. Während der Junge von der Lehrerin wegen seiner , aggressiven' Frage diszipliniert wird, wird das Mädchen in Schutz genommen und erfährt zudem Unterstützung, indem ihr mit Vokabeln ausgeholfen wird. So gelten zweierlei Maßstäbe. Am Ende ironisiert die Lehrerin Knuts Protest, unterstellt ihm, dass er nicht schüchtern sein könne und bestärkt damit noch einmal das Genderstereotyp. Hinter dem Handeln der Lehrerin steht m.E. die Annahme, Mädchen müssten vor Jungen beschützt werden, weil Jungen aggressiv und eben nicht schüchtern sind.

Für Knut stellt sich das Dilemma, dass er entweder gegen diese Unterstellung vehement protestieren kann - und damit die Annahmen seiner Lehrerin über Jungen bestärkt und zusätzlich riskiert, weiter kritisiert zu werden - oder sich angepasst verhält - damit wird aber seine Männlichkeit in Frage gestellt. Das Dilemma verschärft sich dadurch, dass seine Reaktion sowohl auf der geschlechtlichen wie der schulischen Ebene der Bewertung unterliegt.

In allen dargestellten Fällen hängt die Herstellung von Männlichkeit unter Schülern mit einer expliziten oder impliziten Rekurrierung auf Gender durch die Lehrkräfte zusammen. Das heißt einerseits, dass auch in den Interaktionen zwischen Lehrkräften und Schülern Männlichkeit entsteht. Andererseits unterliegt dies negativen Bewertungen, denn konfliktreiches Verhalten von Schülern wird homogenisiert und als problematisches Jungenverhalten etikettiert.

Es ergibt sich für viele Schüler kein erweiterter oder alternativer Interaktionsspielraum, sondern beides - männersolidarisches Verhalten wie Homogenisierung von Jungen als Problemgruppe - führt in den Beispielen eher zu Remaskulinisierungen durch Bezug auf tradierte Elemente des Systems hegemonialer Männlichkeit. Zugespitzt könnte man formulieren, dass die Genderstereotype der Lehrkräfte Männlichkeitsstereotype bei den Schülern befördern. 


\section{Literatur}

Amann, Klaus/Hirschauer, Stefan: Die Befremdung der eigenen Kultur. Frankfurt a. M. 1997

Beaufaÿs, Sandra/Krais, Beate: Doing science - going gender, in: Feministische Studien, Heft 1/2005, S. 82-99

Bohnsack, Ralf: Adoleszenz, Aktionismus und die Emergenz von Milieus, in: Zeitschrift für Sozialisationsforschung und Erziehungssoziologie, Heft 1/1997, S. 318

Brandes, Holger: Der männliche Habitus Band 2, Opladen 2000

Budde, Jürgen: Männlichkeitskonstruktionen in der Institution Schule, in: Zeitschrift für Frauenforschung und Geschlechterstudien, Heft 1/2003, S. 91-101

Budde, Jürgen: Männlichkeit und gymnasialer Alltag, Bielefeld 2005

Budde, Jürgen/Faulstich-Wieland, Hannelore: Chancen und Probleme bei der Dramatisierung von Geschlecht, in: jugendstile, Heft 4/2004, 4, S. 3-8

Connell, Robert: Der gemachte Mann, Opladen 1999

Diefenbach, Heike/Klein, Michael: Bringing Boys Back, in: Zeitschrift für Pädagogik, Heft 6/2001, S. 938-958

Faulstich-Wieland, Hannelore et. al.: Doing Gender im heutigen Schulalltag, Weinheim 2004

Stichweh, Rudolf: Inklusion und Exklusion, Bielefeld 2005

Stürzer, Monika et. al.: Geschlechterverhältnisse in der Schule, Opladen 2003

Zinnecker, Jürgen: Pädagogische Ethnographie, in: Imbke Behnken/Olga Jaumann (Hg.): Kindheit und Schule, Weinheim 1995 